\title{
Neuroinvasive West Nile Virus Presenting as Acute Flaccid Paralysis: A Case Report
}

\author{
Jessica Watari
}

\begin{abstract}
West Nile virus, a mosquito-borne infection, is known to cause a wide spectrum of clinical manifestations ranging from asymptomatic infection to neuroinvasive disease. Advanced age is a known risk factor for developing neuroinvasive disease and also a prognostic indicator of long-term recovery. We present a case of neuroinvasive West Nile virus infection in a 78-year-old man who presented with acute flaccid paralysis and altered mental status. Positive IgM for West Nile virus in the cerebrospinal fluid confirmed the diagnosis. Individuals presenting with meningitis, encephalitis, and/or acute flaccid paralysis should be assessed for West Nile virus especially if presenting during outbreaks in the summer and fall months. Although treatment remains supportive, prompt diagnosis and reporting of the infection can identify potential outbreaks, emphasize the importance of preventative measures, and promote clinician awareness.
\end{abstract}

Keywords: West Nile virus; Neuroinvasive disease; Advanced age; Acute flaccid paralysis

\section{Introduction}

West Nile virus is a mosquito-borne virus that is typically transmitted from infected birds to humans. Infection can manifest from a flu-like illness known as West Nile fever to neuroinvasive disease manifesting as meningitis, encephalitis, and/or acute flaccid paralysis. Diagnosis is made by isolating West Nile virus IgM in the cerebrospinal fluid (CSF). Treatment remains supportive as other therapies such as interferon, ribavirin, and intravenous immunoglobulin continue to be studied. Prognosis of neuroinvasive disease is variable ranging from full recovery to persistent neurological deficits and in some cases fatality.

\section{Case Report}

A 78-year-old man with a past medical history of hyperten-

Manuscript submitted November 15, 2018, accepted November 21, 2018

Rutgers Robert Wood Johnson Medical School, New Brunswick, NJ 08901, USA.Email: jw1097@njms.rutgers.edu

doi: https://doi.org/10.14740/jmc3212 sion and glaucoma presented with a 3-day history of bilateral lower extremity weakness and generalized weakness in August 2016. The lower extremity weakness had progressively become worse over 3 days and he was unable to ambulate. Two weeks prior, he was diagnosed with acute bronchitis and treated with azithromycin. On review of systems, he admitted to subjective fevers, nausea, and vomiting. He denied headache, photophobia, syncope, bowel or bladder incontinence, diarrhea, numbness or tingling in the extremities, sensory changes, recent travel, sick contacts, insect bites, or recent vaccinations. Family history was significant for only hypertension. He denied any toxic habits, reported living alone and was able to perform all activities of daily living prior to admission.

On admission, his temperature was $102.1^{\circ} \mathrm{F}$ with remainder of vital signs stable. Physical examination revealed a lethargic man who was arousable to loud voice. Cardiovascular, respiratory, abdominal, and skin examinations were normal. On neurological examination, cranial nerves II-XII were intact. Muscle strength was $2-3 / 5$ in the left lower distal extremity, $1-2 / 5$ in the right lower distal extremity, and $4 / 5$ in bilateral upper extremities. Sensation to touch was intact in all extremities. Deep tendon reflexes were 0 in bilateral lower extremities and $3+$ in bilateral upper extremities.

Laboratory evaluation was significant for hyponatremia (131 mEq/L, normal 135 - $145 \mathrm{mEq} / \mathrm{L}$ ) and elevated creatinine kinase (787 units/L, normal 22 - 198 units/L). Complete blood count and comprehensive metabolic panel were within normal limits. Urinalysis showed $1+$ ketone, $1+$ protein, $2+$ blood, and negative nitrite and leukocyte esterase. Hepatitis B, hepatitis $\mathrm{C}$, cytomegalovirus, rapid plasma reagin (RPR), and HIV testing were negative. Chest radiograph and computed tomography (CT) of the head showed no acute findings. Lumbar puncture was performed. CSF was colorless and clear with glucose $58 \mathrm{mg} / \mathrm{dL}$, elevated protein $(79 \mathrm{mg} / \mathrm{dL}$, normal 15 - 45 $\mathrm{mg} / \mathrm{dL})$, elevated red blood cell count $(41$ cells $/ \mu \mathrm{L}$, normal $<$ 1 cells $/ \mu \mathrm{L})$, and elevated white blood cell count $(41$ cells $/ \mu \mathrm{L}$, normal 0 - 5 cells $/ \mu \mathrm{L}$ ) with differential of $6 \%$ neutrophils, $92 \%$ lymphocytes, and $2 \%$ monocytes. CSF gram stain showed no white blood cells and no organisms.

The patient was empirically treated for meningoencephalitis with ampicillin, ceftriaxone, acyclovir, and vancomycin. In the following 3 days, he continued to be febrile. Plasmapheresis was started on hospital day 3 for presumed diagnosis of Guillain-Barre syndrome. Neurological examination remained unchanged. He subsequently received five additional rounds of plasmapheresis. CSF cultures and blood cultures returned with no growth for 5 days. CSF analysis showed negative IgG and 
IgM for Lyme disease, negative Venereal Disease Research Laboratory (VDRL), negative herpes simplex virus-1 and herpes simplex virus-2, and positive IgM and IgG for West Nile virus. The patient was diagnosed with neuroinvasive West Nile virus. Antibiotics, acyclovir, and plasmapheresis were discontinued and supportive measures were continued.

\section{Discussion}

West Nile virus is a mosquito-borne virus flavivirus [1] that was first isolated from the West Nile province of Uganda in 1937 [2]. In 1999, the virus was detected in the United States with 59 patients hospitalized and $63 \%$ showing clinical signs of encephalitis. During the outbreak, seven patients died with advanced age being an independent risk factor for death [3]. West Nile virus subsequently spread to the contiguous United States, South Caribbean, and Canada [4]. Although transmission primarily occurs from infected birds to humans via a mosquito vector, other potential modes of transmission including blood transfusions [5], organ transplants [6], and congenital infection [7] have been reported.

Approximately $80 \%$ of individuals infected with West Nile virus will be asymptomatic. Majority of individuals who do become symptomatic will develop an acute febrile illness known as West Nile fever. Presenting signs and symptoms include fever, headache, fatigue, myalgias, nausea, vomiting, and a generalized maculopapular rash [8]. In less than $1 \%$ of cases, the virus may penetrate the blood-brain barrier and directly invade the neurons resulting in neuroinvasive disease manifesting as meningitis, encephalitis, or acute flaccid paralysis [9]. Advanced age, male sex, hypertension, and diabetes mellitus have been identified as potential risk factors for developing neuroinvasive disease [10].

West Nile meningitis presents similarly to other viral meningitides with fever, headache, and meningeal signs, whereas West Nile encephalitis ranges from a self-limiting confusional state to severe encephalopathy and coma [8]. Acute flaccid paralysis may present alone or concomitantly with meningitis and encephalitis. Patterns of weakness described include acute monoplegia, asymmetric upper and lower extremity weakness, and generalized asymmetric quadriplegia. Poliomyelitis-like syndrome with motor axonopathy and/or anterior horn cell involvement and Guillain-Barre-like syndrome with demyelinating sensorimotor neuropathy have also been described [11].

Diagnosis of West Nile virus infection is made by detecting IgM antibody in the serum or CSF using IgM antibodycapture enzyme-linked immunosorbent assay (MAC-ELISA). The presence of IgM in the CSF is indicative of neuroinvasive West Nile virus as IgM is unable to cross the blood-brain barrier. The majority of patients with neuroinvasive disease will have detectable IgM present in the CSF within 8 days of symptom onset. CSF analysis will typically show normal glucose, elevated protein $(<150 \mathrm{mg} / \mathrm{dL})$, and pleocytosis $(<500$ cells/ $\mathrm{uL}$ ) usually with lymphocyte predominance [12]. Additional laboratory findings of hyponatremia and elevated creatinine kinase have been reported. Treatment for West Nile virus disease remains supportive with pain control, antiemetics, rehydration, monitoring for elevated intracranial pressure, and seizure control if needed [9]. Potential therapies including interferon, ribavirin, and intravenous immunoglobulin have been investigated and have either been found to be non-efficacious or remain to be in the early stages of development $[9,13,14]$.

The prognosis of West Nile virus disease is variable. According to the Centers for Disease Control and Prevention, from 1999 - 2015 the fatality rate of non-neuroinvasive West Nile virus infection was $<1 \%$ while the fatality rate for neuroinvasive disease was 9\% [15]. Fatigue, headache, and myalgias have been reported as persistent symptoms in subjects with neuroinvasive disease [16]. Individuals with acute flaccid paralysis have variable improvement with one study cohort showing varying degrees of improvement at 4-month follow-up and another cohort showing no improvement in limb strength at 8-month follow-up [11, 16].

Given the morbidity and mortality of neuroinvasive West Nile virus infection, it is important to consider it in the differential diagnosis for patients presenting with neurological symptoms including meningeal signs, altered mental status, and/or acute paralysis. Heightened awareness of West Nile virus especially during the summer and early fall months is vital in order to diagnose this clinically variable disease.

\section{Financial Disclosures}

The author has no financial disclosures relevant to this manuscript.

\section{Disclosure Statement}

The author declares that there is no conflict of interest regarding the publication of this paper.

\section{References}

1. Brinton MA. The molecular biology of West Nile Virus: a new invader of the western hemisphere. Annu Rev Microbiol. 2002;56:371-402.

2. Campbell B, Good RA. Antigen-antibody mechanisms in neurotropic virus diseases. Ann Allergy. 1949;7(4):471481.

3. Nash D, Mostashari F, Fine A, Miller J, O'Leary D, Murray $\mathrm{K}$, Huang $\mathrm{A}$, et al. The outbreak of West Nile virus infection in the New York City area in 1999. N Engl J Med. 2001;344(24):1807-1814.

4. Gray TJ, Webb CE. A review of the epidemiological and clinical aspects of West Nile virus. Int J Gen Med. 2014;7:193-203.

5. Pealer LN, Marfin AA, Petersen LR, Lanciotti RS, Page PL, Stramer SL, Stobierski MG, et al. Transmission of West Nile virus through blood transfusion in the United States in 2002. N Engl J Med. 2003;349(13):1236-1245.

6. Nett RJ, Kuehnert MJ, Ison MG, Orlowski JP, Fischer M, 
Staples JE. Current practices and evaluation of screening solid organ donors for West Nile virus. Transpl Infect Dis. 2012;14(3):268-277.

7. O'Leary DR, Kuhn S, Kniss KL, Hinckley AF, Rasmussen SA, Pape WJ, Kightlinger LK, et al. Birth outcomes following West Nile Virus infection of pregnant women in the United States: 2003-2004. Pediatrics. 2006;117(3):e537-545.

8. Sejvar JJ. Clinical manifestations and outcomes of West Nile virus infection. Viruses. 2014;6(2):606-623.

9. Debiasi RL, Tyler KL. West Nile virus meningoencephalitis. Nat Clin Pract Neurol. 2006;2(5):264-275.

10. Jean CM, Honarmand S, Louie JK, Glaser CA. Risk factors for West Nile virus neuroinvasive disease, California, 2005. Emerg Infect Dis. 2007;13(12):1918-1920.

11. Sejvar JJ, Bode AV, Marfin AA, Campbell GL, Ewing D,
Mazowiecki M, Pavot PV, et al. West Nile virus-associated flaccid paralysis. Emerg Infect Dis. 2005;11(7):10211027.

12. Petersen LR, Brault AC, Nasci RS. West Nile virus: review of the literature. JAMA. 2013;310(3):308-315.

13. Chowers MY, Lang R, Nassar F, Ben-David D, Giladi M, Rubinshtein E, Itzhaki A, et al. Clinical characteristics of the West Nile fever outbreak, Israel, 2000. Emerg Infect Dis. 2001;7(4):675-678.

14. Agrawal AG, Petersen LR. Human immunoglobulin as a treatment for West Nile virus infection. J Infect Dis. 2003;188(1):1-4.

15. Sejvar JJ, Haddad MB, Tierney BC, Campbell GL, Marfin AA, Van Gerpen JA, Fleischauer A, et al. Neurologic manifestations and outcome of West Nile virus infection. JAMA. 2003;290(4):511-515. 